\title{
Dynamic Response Analysis of Simply Supported Steel Beam Under Natural Fire Field Load
}

\author{
Tang Feng, Yang Jinglin*, Wan Linhui, Ou Yangguoyuan \\ College of Engineering, Hunan Agricultural University, Changsha, China \\ Email address: \\ 21134293@qq.com (Tang Feng), yangjl@hunau.edu.cn (Yang Jinglin) \\ ${ }^{*}$ Corresponding author
}

\section{To cite this article:}

Tang Feng, Yang Jinglin, Wan Linhui, Ou Yangguoyuan. Dynamic Response Analysis of Simply Supported Steel Beam Under Natural Fire Field Load. Science Discovery. Vol. 5, No. 7, 2017, pp. 603-608. doi: 10.11648/j.sd.20170507.32

Received: November 13, 2017; Accepted: November 23, 2017; Published: January 7, 2018

\begin{abstract}
The outbreak of fire has a great influence on. the carrying capacity of structure. the elastic modulus, flexural rigidity, yield strength and natural frequency of structural steel are discussed on the effects of fire temperature in this paper. The simply supported steel beam to form a plastic hinge when the ultimate moment changes in the first phase of the beam movement and the dynamic responses of simply supported steel beams under different loads were analyzed. Specific to the Q235 H-shaped steel case of $\mathrm{H}$-shaped steel was calculated in high temperature structural steel bending stiffness, yield strength and natural frequency of simply supported steel beam in the two stages of movement of the dynamic response is analyzed. The analysis showed that the fire duration was $2.5 \mathrm{~h}$, the yield strength of steel is only $85.63 \mathrm{~N} / \mathrm{mm}^{2}$, a drop of $63.56 \%$, while the component in the fire field in two phases dynamic deflection is also rapid growth, an increase of over 50\%, almost lost capacity, in addition to members of this excessive plastic deformation is a serious cause of the damaged component. Dangerous. Therefore, in order to ensure the safety of steel structure design, the adverse effects should be taken into full consideration in the design of simply supported steel beams.
\end{abstract}

Keywords: Fire Field, Simply Supported Steel Beam, Bearing Capacity, Ultimate Bending Moment, Dynamic Response

\section{火灾荷载作用下简支钢梁动力响应分析}

汤峰, 杨敬林 ${ }^{*}$, 万琳辉, 欧阳国元

工学院, 湖南农业大学, 长沙, 中国

邮箱

21134293@qq.com（汤峰）, yangj1@hunau.edu.cn（杨敬林）

摘要：火灾爆发对结构承载能力有较大的影响，本文根据自然火灾场不同时刻钢构件的升温变化，讨论了火灾高温对结 构钢弹性模量、抗弯刚度、屈服强度和自振频率的影响, 分析了简支钢梁在第一相运动中梁跨中形成一个塑性铰时的极 限弯矩变化，同时对简支钢梁在火灾场冲击波荷载作用下在不同阶段不同时刻的动力响应进行了分析。具体以Q235工字 型钢梁为例计算了工字型钢梁在火灾高温下结构钢的抗弯刚度、屈服强度和自振频率，对简支钢梁在两个阶段运动时的 动力响应进行了分析，分析表明在火灾持续时间达 $2.5 \mathrm{~h}$ 时，钢构件的屈服强度仅为 $85.63 \mathrm{~N} / \mathrm{mm}^{2}$, 降幅达 $63.56 \%$ ，同时构 件在火灾场中两个阶段动挠度也增长较快, 增幅超 $50 \%$, 几乎丧失承载力, 此外钢构件这种过大的塑性变形也严重会导 致构件损坏, 出现危险。因此, 在简支钢梁工程设计中, 要充分考虑这种不利影响, 以保证钢结构设计安全。

关键词: 火灾场, 简支钢梁, 承载能力, 极限弯矩, 动力响应 


\section{1. 引言}

火灾场的钢结构在某些特殊环境中, 要承受由于火灾 引起的冲击荷载而发生运动, 钢结构构件弹性模量、抗弯 刚度和屈服强度都会随着火灾温度的上升而变化, 导致构 件变形较大, 甚至破坏[1]。余同希等[2]人分别对冲击荷载 作用下的理想弹塑性梁的动力响应进行了一些理论分析, 杨桂通[3]对结构在冲击荷载作用下进行了实验研究。李国 强[4]等在钢结构构件升温过程中对钢材的材料特性作了较 为详细的论述。由于火灾下当钢结构构件温度升高时, 钢 构件要发生膨胀对结构变形产生影响, 当这种膨胀和变形 受到约束时, 构件还会产生附加内力。基于火灾场引起钢 构件变形, 火灾高温对钢材的弹性模量、抗弯刚度、塑性 弯矩和极限承载力都有影响, 本文研究简支钢梁在自然火 灾场中的动力响应, 并通过具体实例探讨了简支钢梁在火 灾场中第一相运动和第二相运动的动力挠度 [5], 得出结论。

如图1所示。在自然火灾场中的等截面简支钢梁看作 是理想的弹塑性体, 跨长为 $l$, 质量密度为 $m$, 受到自然火 灾冲击波作用 [6-8]。冲击波荷载的主要特征是在短时间内 上升到峰值, 随即迅速减到零, 然后有数值不大的负相压 力[9-11]。假定火灾冲击波按下列线规律来近似描述

$$
p(t)=p_{0}\left(1-\frac{t}{\theta}\right)
$$

式中, $\theta$ 为正相压力持续时间。

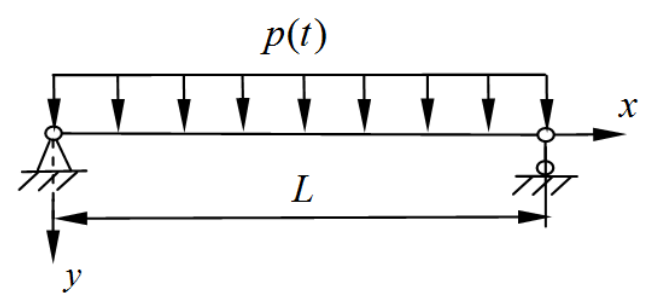

图1 火灾场简支钢梁简化模型。

\section{2. 火灾场中简支钢梁的动力响应分析}

\section{1. 火灾场高温下结构钢的力学参数}

目前国内尚没有系统的结构钢在高温下的材料性质 的实验资料, 为保守起见, 国内钢结构抗火分析一般均采 用ECCS所采用的方案。ECCS给出的高温下结构钢屈服强 度公式为

$$
\begin{aligned}
\frac{f_{y T}}{f_{y}} & =1+\frac{T_{s}}{767 \ln \frac{T_{s}}{1750}} \quad 0 \leq T_{s} \leq 600^{\circ} \mathrm{C} \\
\frac{f_{y T}}{f_{y}} & =\frac{108\left(1-\frac{T_{s}}{1000}\right)}{T_{s}-440} \quad 600 \leq T_{s} \leq 1000^{\circ} \mathrm{C}
\end{aligned}
$$

式中, $f_{T y}$ 为火灾高温下结构钢屈服强度, $f_{y}$ 为常温下 结构钢屈服强度, $T_{s}$ 为火灾高温下结构钢的绝对强度, $T_{s}$
取钢构件在ISO834标准升温条件下的温度, 令 $T_{g}=20^{\circ} \mathrm{C}$, $\mathrm{B}$ 为截面保护层参数 $B=\frac{\lambda_{i}}{d_{i}} \cdot \frac{F_{i}}{V}, \lambda_{i}$ 为保护层的导热系数, $d_{i}$ 为保护层厚度, $\frac{F_{i}}{V}$ 为截面形状系数, 均查表确定。

$$
T_{s}=\left(\sqrt{0.044+5 \times 10^{-5} B}-0.2\right) t+T_{g}(0) \quad\left(0 \leq T_{s} \leq 600^{\circ} \mathrm{C}\right)
$$

钢材初始弹性模量 $E_{T}$ 采用的方案为

$$
\begin{aligned}
\frac{E_{T}}{E} & =1-17.2 \times 10^{-12} T_{s}^{4}+11.8 \times 10^{-9} T_{s}^{3} \\
& -34.5 \times 10^{-7} T_{s}^{2}+15.9 \times 10^{-5} T_{s} \\
& \left(0 \leq T_{s} \leq 600^{\circ} \mathrm{C}\right)
\end{aligned}
$$

式中, $E$ 为常温下结构钢弹性模量, $\mathrm{N} / \mathrm{mm}^{2}, E_{T}$ 为火 灾高温 $T_{s}$ 时结构钢初始弹性模量。

\section{2. 火灾场中简支钢梁的动力响应}

\subsection{1. 第一相运动}

简支钢梁在火灾场中第一相的运动是弹性的, 其运动 方程为

$$
E_{T} I w^{(4)}+m \ddot{w}=p(t)
$$

设将梁的动力挠度分为两部分 $w=w_{1}+w_{2}$, 其中 $w_{1}$ 是静态挠度, 它满足

$$
E_{T} I w_{1}^{(4)}=p(t)
$$

$W_{2}$ 满足下式

$$
E_{T} I w_{2}^{(4)}+m \ddot{w}_{2}=-m \ddot{w}_{1}
$$

简支钢梁的边界条件为

$$
\begin{aligned}
& x=0, x=l, w_{1}=w_{2}=0 \\
& x=0, x=l, w_{1}^{\prime \prime}=w_{2}^{\prime \prime}=0
\end{aligned}
$$

梁第一相运动规律的解为

$$
\begin{aligned}
& w(x, t)=\frac{4 p_{0} l^{4}}{\pi^{5} E_{T} I} \sum_{n=1,3, \cdots}^{\infty} \frac{1}{n^{5}}\left(1-\frac{t}{\theta}+\frac{\sin \omega_{n} t}{\theta \omega_{n}}-\cos \omega_{n} t\right) \sin \frac{n \pi x}{l} \\
& \dot{w}(x, t)=\frac{4 p_{0} l^{4}}{\pi^{5} E_{T} I} \sum_{n=1,3, \cdots}^{\infty} \frac{1}{n^{5}}\left(-\frac{1}{\theta}+\frac{\cos \omega_{n} t}{\theta \omega_{n}}+\sin \omega_{n} t\right) \sin \frac{n \pi x}{l} \\
& M(x, t)=\frac{4 p_{0} l^{4}}{\pi^{3}} \sum_{n=1,3, \cdots}^{\infty} \frac{1}{n^{3}}\left(1-\frac{t}{\theta}+\frac{\sin \omega_{n} t}{\theta \omega_{n}}-\cos \omega_{n} t\right) \sin \frac{n \pi x}{l} \\
& Q(x, t)=\frac{4 p_{0} l}{\pi^{2}} \sum_{n=1,3, \cdots}^{\infty} \frac{1}{n^{2}}\left(-\frac{t}{\theta}+\frac{\sin \omega_{n} t}{\theta \omega_{n}}-\cos \omega_{n} t\right) \cos \frac{n \pi x}{l}
\end{aligned}
$$


式中, $\omega_{n}$ 为简支梁的自振频率, 由下式确定

$$
\omega_{n}=\left(\frac{n \pi}{l}\right)^{2} \sqrt{\frac{E_{T} I}{m}} \quad(n=1,2,3, \cdots)
$$

\subsection{2. 第二相运动}

当梁第一相运动一直延续到跨中点的弯矩到达极限 值 $M_{0}$, 在该处形成塑性铰后, 梁即进入第二相运动。此后 $\left(t>t_{1}\right)$, 假定梁的运动是在第一相末的挠度再叠加一刚性转 动。

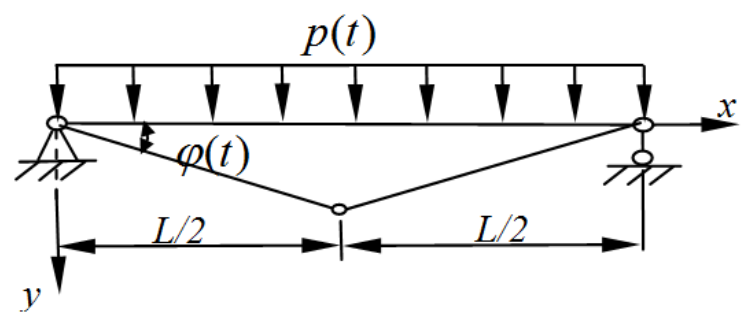

图2 火灾场简支钢梁第二相运动。

$$
w(x, t)=\varphi(t) x+w_{0} \sin \frac{\pi x}{l} \quad\left(0 \leq x \leq \frac{l}{2}\right)
$$

式中, $w_{0}=\frac{4 p_{0} l^{4}}{\pi^{5} E_{T} I}\left(1-\frac{t_{1}}{\theta}+\frac{\sin \omega_{1} t_{1}}{\theta \omega_{1}}-\cos \omega_{1} t_{1}\right)$ 为简支钢 梁第一相运动持续到梁形成塑性铰 $t=t_{1}$ 时的最大挠度。式 (7)表明第二相运动的时间是从 $t_{1}$ 开始计算的，总时刻是 $t+t_{1}$ 。梁在第二相运动中假设梁只做刚性转动。梁在第二 相运动开始时载荷尚未卸去, 即 $t_{1}<\theta$, 则有

$$
p(t)=p_{0}\left(1-\frac{t+t_{1}}{\theta}\right)
$$

梁在第二相运动微分方程为

$$
\frac{m l^{3}}{24 M_{p}} \ddot{\varphi}(t)=1-\frac{t+t_{1}}{\theta}-\frac{M_{0}}{M_{p}}
$$

式中, $M_{p}=\frac{1}{8} p l^{2}, M_{0}=\frac{4 p_{0} l^{2}}{\pi^{3}}\left(1-\frac{t_{1}}{\theta}+\frac{\sin \omega_{1} t_{1}}{\theta \omega_{1}}-\cos \omega_{1} t_{1}\right)$ 为 梁在第一相运动形成塑性铰时的极限弯矩。
式(8)解为

$$
w_{\max }=w_{0}+\frac{\varphi_{\max } l}{2}=\frac{M_{0} l^{2}}{9.6 E_{T} I}\left(1+0.049 \frac{\omega_{1}^{2} \theta^{2}}{M_{0} / M_{p}} \eta\right)
$$

$$
\begin{gathered}
\text { 式中, } \varphi_{\max }=\frac{M_{p} \theta^{2}}{m l^{3}} \eta \\
\eta=\left[\frac{17.33 \gamma}{\theta \omega_{1}}+8\left(\frac{M_{0}}{M_{p}}-1+\frac{t_{1}}{\theta}\right)^{2}\right] \times \sqrt{\left(\frac{M_{0}}{M_{p}}-1+\frac{t_{1}}{\theta}\right)^{2}+\frac{2.17 \gamma}{\theta \omega_{1}}} \\
-\left(\frac{M_{0}}{M_{p}}-1+\frac{t_{1}}{\theta}\right)^{3}-\frac{26 \gamma\left(M_{0} / M_{p}-1+t_{1} / \theta\right)}{\theta \omega_{1}} \\
\gamma=\sin \omega_{1} t_{1}+\frac{1}{\theta t_{1}}\left(1-\cos \omega_{1} t_{1}\right)
\end{gathered}
$$

\section{3. 实例数值分析}

如图3所示, 某双轴对称工字型简支钢梁, 跨度为 $9 \mathrm{~m}$, 钢梁材料为Q235, 常温下屈服强度为 $f_{y}=235 \mathrm{MPa}$, 钢材 外表面采用厚涂型钢结构防火涂料, 厚度为 $40 \mathrm{~mm}$, 密度 $\rho_{i}=300 \mathrm{~kg} / \mathrm{m}^{3}$, 导热系数 $\lambda_{i}=0.11 \mathrm{~W} /\left(\mathrm{m}^{\circ} \mathrm{C}\right)$, 钢梁耐火极

\begin{tabular}{|c|c|c|c|c|c|}
\hline 火灾燃烧时间 $\mathbf{t}(\mathbf{h}$ 或s) & 构件升温温度 $T\left({ }^{\circ} \mathrm{C}\right)$ & 弹性模量 $\boldsymbol{E}_{T}\left(\times 10^{5} \mathrm{~N} / \mathrm{mm}^{2}\right)$ & 刚度 $E_{T} I\left(\times 10^{5} \mathrm{~N} \cdot \mathrm{mm}^{2}\right)$ & 屈服强度 $f_{y} r\left(\mathrm{~N} / \mathrm{mm}^{2}\right)$ & 自振频率 $\omega_{1}\left(\times 10^{-2} \mathrm{~Hz}\right)$ \\
\hline $0 / 0$ & 20 & 2.0037 & 2.0076 & 233.63 & 10.0168 \\
\hline $0.25 / 900$ & 73.76 & 1.9943 & 1.9887 & 227.86 & 9.9933 \\
\hline $0.5 / 1800$ & 127.52 & 1.9682 & 1.9369 & 220.08 & 9.9275 \\
\hline $0.75 / 2700$ & 181.28 & 1.9343 & 1.8708 & 210.50 & 9.8417 \\
\hline $1 / 3600$ & 235.04 & 1.8950 & 1.7955 & 199.13 & 9.7412 \\
\hline $1.25 / 4500$ & 288.80 & 1.8455 & 1.7029 & 185.89 & 9.6131 \\
\hline $1.5 / 5400$ & 342.56 & 1.7742 & 1.5739 & 170.65 & 9.4256 \\
\hline $1.75 / 6300$ & 396.32 & 1.6627 & 1.3822 & 153.24 & 9.1245 \\
\hline $2 / 7200$ & 450.08 & 1.4855 & 1.1033 & 133.45 & 8.6245 \\
\hline $2.25 / 8100$ & 503.84 & 1.2103 & 0.7324 & 111.02 & 7.7849 \\
\hline $2.5 / 9000$ & 557.60 & 0.7980 & 0.3184 & 85.63 & 6.3214 \\
\hline
\end{tabular}
限 $2.5 \mathrm{~h}$ 。此实例计算中取 $n=1, p_{0}=300 \mathrm{kN} / \mathrm{m}$, 正相压力持 续时间 $\theta=7 \mathrm{~s}, t_{1}=4 \mathrm{~s}$, 分析火灾场中简支钢梁在各时刻的温 度、弹性模量、刚度、屈服强度和自振频率的变化, 在此 基础上分别计算了 $t=1.5 \mathrm{~h}$ 和 $t=2.5 \mathrm{~h}$ 时简支钢梁的动力响应。 计算结果分别列于表1和图4至图8中。

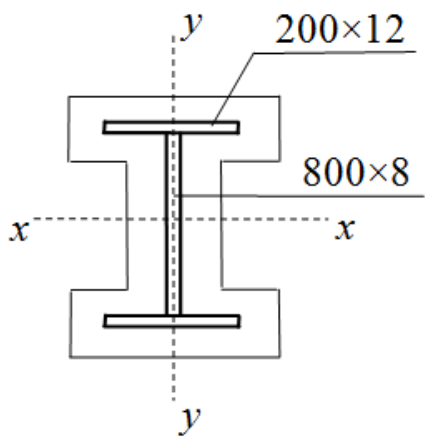

图3 钢梁工字型横截面图。

表1 火灾场简支钢梁材料参数计算结果。 


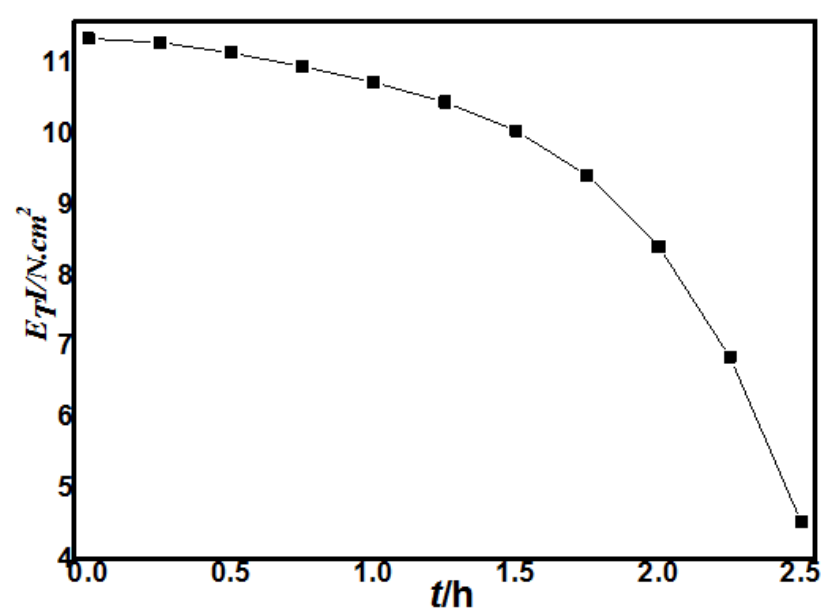

图4 火灾场梁刚度 $E_{T} I$ 变化。

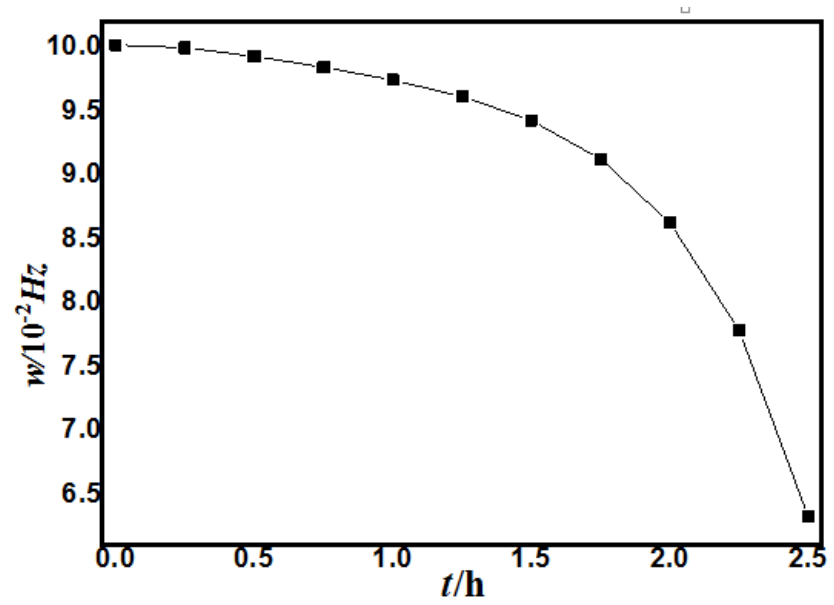

图5 火灾场梁自振频率 $\omega_{1}$ 变化。

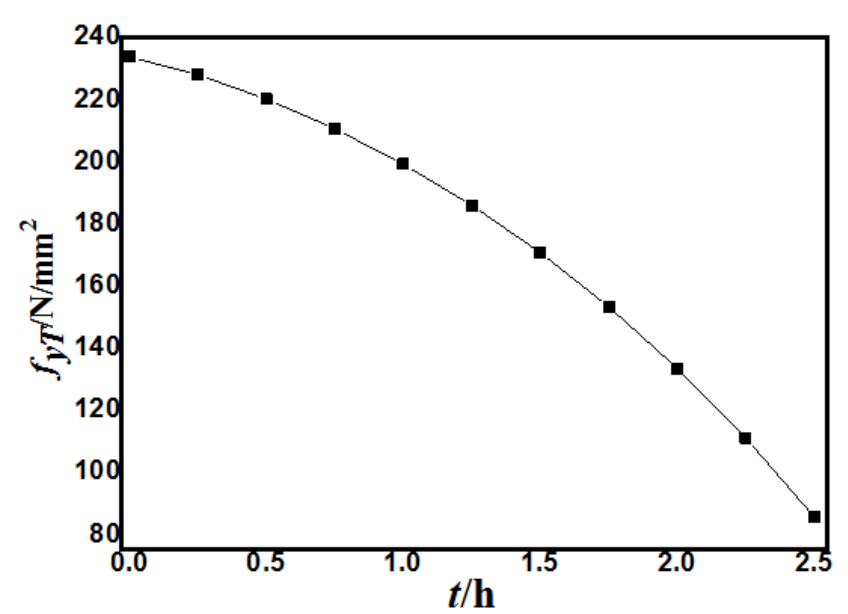

图6 火灾场梁屈服强度 $f_{y T}$ 变化。

表1和图4至图6分别表示火灾场中简支钢梁抗弯刚 度、自振频率和屈服强度的变化关系。从上述图表上可 以看出, 钢梁在自然火灾高温情况下, 钢构件的温度呈 直线上升, 构件的抗弯刚度和自振频率都下降, 在火灾 持续时间 $1.5 \mathrm{~h}$ 后几乎呈直线下降，降幅接近 $50 \%$ 。在火 灾场简支钢梁升温过程中构件的屈服强度降幅最大, 钢
构件的屈服强度在火灾场 $0.5 \mathrm{~h}$ 以内下降速度不大, 在 $1.5 \mathrm{~h}$ 左右升温至 $300^{\circ} \mathrm{C}$ 以上后, 构件屈服强度为 $170.653 \mathrm{~N} / \mathrm{mm}^{2}$, 下降了 $27.38 \%, 2.5 \mathrm{~h}$ 后构件温度升温至 接近 $600{ }^{\circ} \mathrm{C}$, 钢构件的屈服强度仅为 $85.63 \mathrm{~N} / \mathrm{mm}^{2}$, 降幅 达 $63.56 \%$, 钢构件承载力几乎丧失。

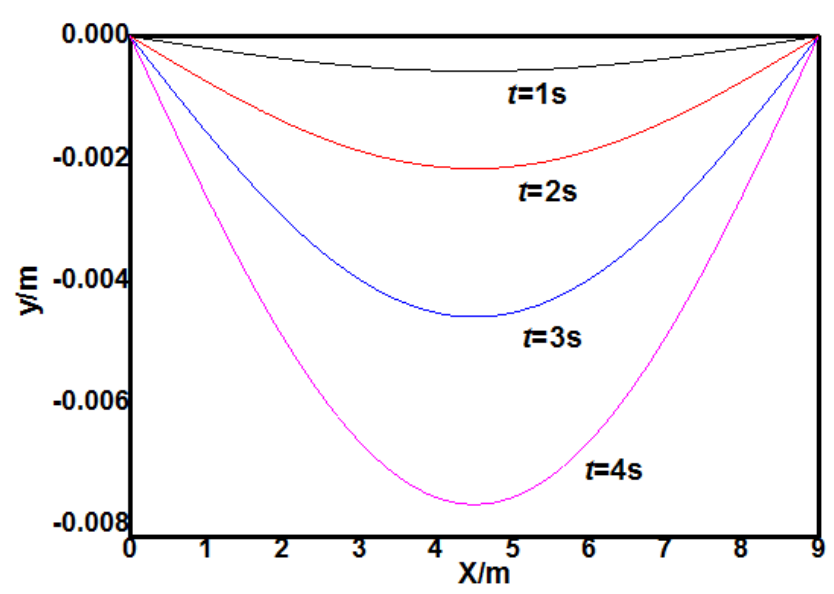

(a) 火灾燃烧时间至 $1 \mathrm{~h}$

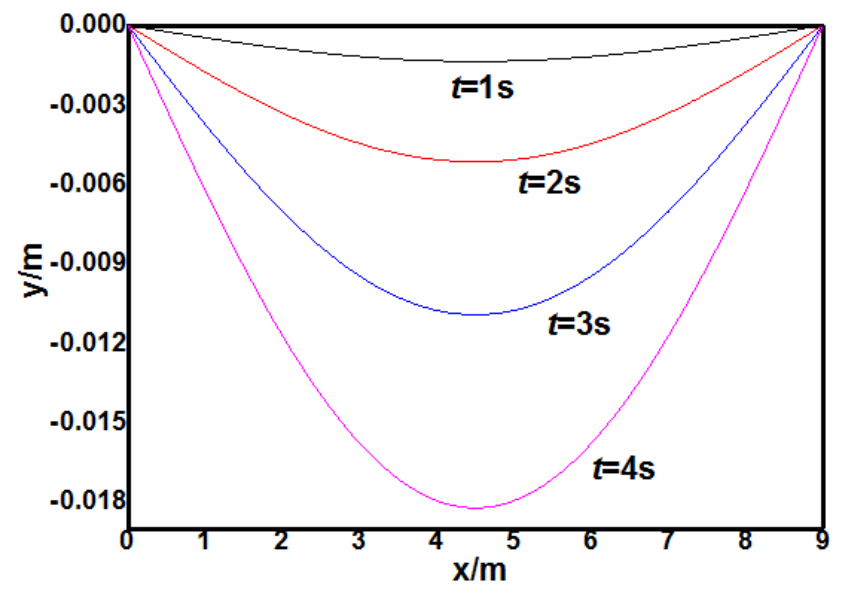

(b) 火灾燃烧时间至 $2.5 \mathrm{~h}$

图7 火灾场第一相简支梁动挠度。

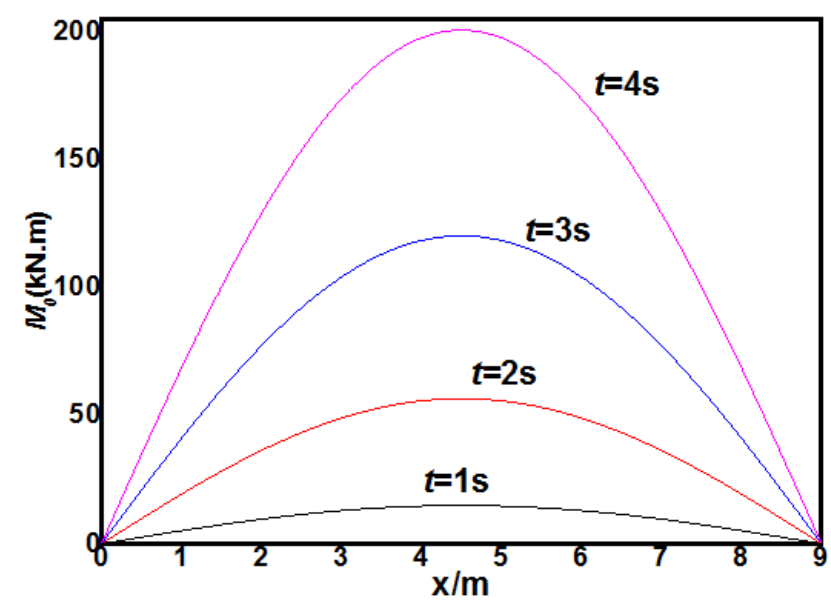

图8 火灾场第一相简支梁形成一个塑性铰时极限弯矩。 


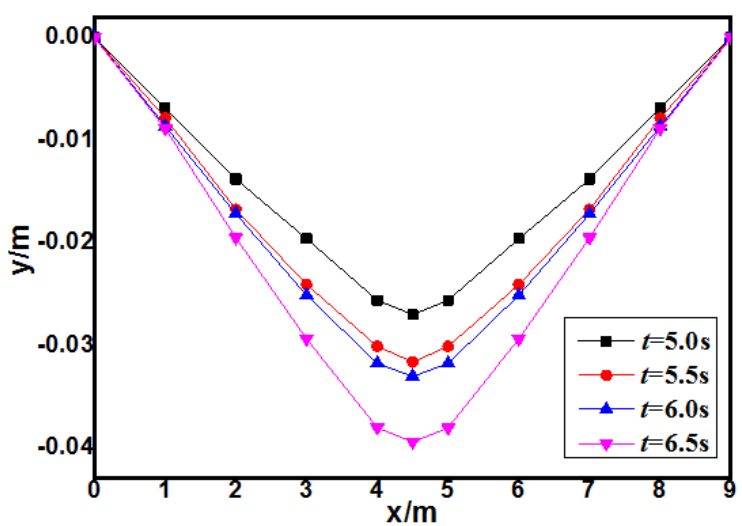

(a)火灾燃烧时间至 $1 \mathrm{~h}$

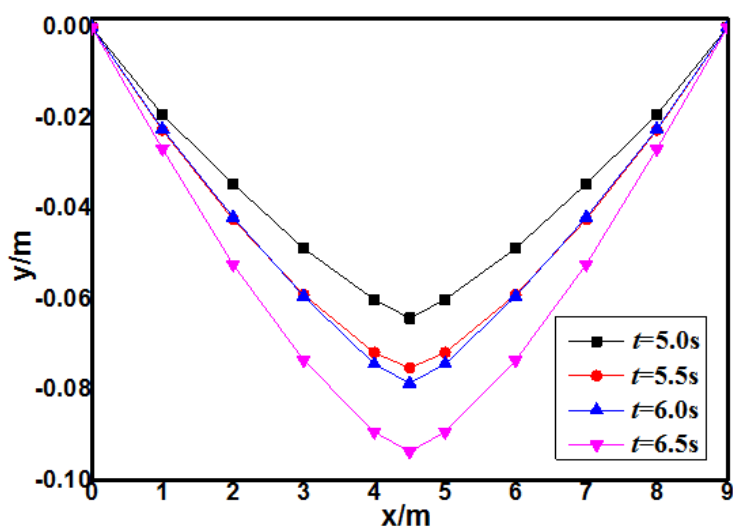

(b)火灾燃烧时间至2.5h

图9 火灾场第二相简支梁动挠度。

由图7至图9可以看出, 简支钢梁第一相运动处于弹性 阶段, 随着温度升高钢梁弹性模量逐渐减少, 但在火灾场 不同时刻受到火灾冲击波荷载作用动挠度逐渐增大, 尤以 在跨中达最大值, 当火灾燃烧时间至 $1 \mathrm{~h}$ 最大动挠度接近 $0.008 \mathrm{~m}$, 当当火灾燃烧时间至 $2.5 \mathrm{~h}$ 最大动挠度迅速增大至 约 $0.018 \mathrm{~m}$ 。另外, 图 8 表明简支梁在第一相运动形成一个 塑性铰时极限弯矩也在跨中达最大, 最大极限弯矩接近 $200 \mathrm{kN} \cdot \mathrm{m}$ 。在图 10 中，简支钢梁在中点形成塑性铰后即进 入第二相运动, 此时简支钢梁的动挠度随火灾燃烧时间的 延长增大非常快, 火灾燃烧至 $1 \mathrm{~h}$ 时跨中动挠度才 $0.04 \mathrm{~m}$, 当燃烧至 $2.5 \mathrm{~h}$ 时, 钢梁动挠度迅速增至接近 $0.10 \mathrm{~m}$, 增幅 达 $50 \%$ 以上, 已远远超过受弯构件的挠度允许值, 这种过 大的塑性变形严重会导致结构破坏, 是很不利的。

\section{4. 结论}

本文分析了简支钢梁在火灾场冲击波荷载作用下的 动力响应, 探讨了简支钢梁在火灾场不同时刻火灾高温时 的抗弯刚度、屈服强度和梁自振频率的变化规律。自然火 灾发生后, 简支钢梁的抗弯刚度、屈服强度和自振频率均 迅速下降, 屈服强度下降速度最快, 降幅也最大。在自然 火灾场不同时刻钢构件升温变化后高温对结构钢弹性模 量、抗弯刚度的影响变化, 并对简支钢梁在火灾场冲击波 荷载作用下在不同阶段不同时刻的动力响应进行了讨论。
简支钢梁在第一相运动中处于弹性阶段，火灾场钢构 件升温后，动挠度和极限弯矩逐渐增大，燃烧时间越长， 跨中动挠度增幅越快, 直至钢构件几乎丧失承载力。火灾 场第二相运动中处于弹塑性阶段和塑性阶段, 塑性变形急 剧增大, 燃烧时间至 $2.5 \mathrm{~h}$ 左右, 钢构件由于过大的塑性变 形严重导致构件破坏。因此，在简支钢梁设计时，自然火 灾会导致钢构件的弹性模量、屈服强度、自振频率急剧减 小, 在工程实际中要充分考虑这些因素的影响, 以减小损 失。在实例分析中具体以Q235工字型钢梁为例计算了工字 型钢梁在火灾高温下结构钢的抗弯刚度、屈服强度和自振 频率, 对简支钢梁在两个阶段运动时的动力响应进行了分 析，分析表明在火灾持续时间达 $2.5 \mathrm{~h}$ 时，钢构件的屈服强 度仅为 $85.63 \mathrm{~N} / \mathrm{mm}^{2}$, 降幅达 $63.56 \%$, 同时构件在火灾场中 两个阶段动挠度也增长较快, 增幅超 $50 \%$, 几乎丧失承载 力, 此外钢构件这种过大的塑性变形也严重会导致构件损 坏, 出现危险。因此, 在钢结构设计中必需要考虑火灾作 用。

基金项目

湖南省科技计划项目（2011NK3070）。

\section{参考文献}

[1] 李国强, 蒋首超, 林桂祥. 钢结构抗火计算与设计 [M]. 北京: 中国建筑工业出版社, 1999.6:75-102。

[2] 余同希. 冲击荷载作用下材料和结构的响应[J]. 力学进展, 1997.27(2):276-280。

[3] 杨桂通. 塑性动力学 [M]. 北京: 高等教育出版社, 2000:93-102。

[4] 杨桂通. 结构在冲击荷载作用下的实验研究 $[\mathrm{J}]$. 力学学报, 1990.22(3):374-379。

[5] Yu T X, Yang J L Reid S R. Dynamic behavior of elastic-plastic free-free beams subjected to impulsive loading Int J Solids Structures [J]. 1996, 33:2659-2680.

[6] 王振清, 韩玉来, 王永军, 苏娟. 火灾场冲击波荷载作用下 简支钢梁动力响应 [J]. 振动与冲击, 2007.26(4):69-72,170。

[7] 赵启超, 孙胜. 火灾场冲击波荷载作用下刚塑性简支钢梁的 残余变形 $[J]$. 黑龙江科技信息, 2011,7(7):314。

[8] 王振清, 韩玉来, 苏娟, 乔牧. 钢筋混凝土梁火灾承载力及 等效楼面荷载分析[J]. 武汉理工大学学报, 2007.29(6):65-68。

[9] GB50017-2003钢结构设计规范[S]. 北京: 中国计划出版社, 2003。

[10] 朱婉婉. 通风系数对钢构件的耐火性能影响研究 [J]. 武警学 院学报, 2016,32(8):9-12。

[11] 张之峰, 石国峰. 受火钢结构温度-结构耦合场分析 [J]. 电脑 知识与技术, 2015,11(2):260-262。 


\section{作者简介}

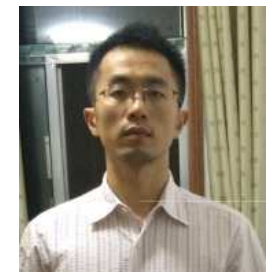

汤峰（1982-), 男, 硕士研究生, 主 要从事砌体结构、土木工程施工等研 究工作, 国家一级建造师, 参与编写

《CECS 256-2009蒸压粉煤灰砖建筑 技术规范》等行业规范。 